\title{
Influência da lista única de uma regional de transplantes de córnea em um Banco de Olhos vinculado a um hospital escola
}

\author{
Influence of a single regional list for corneal transplantation on the eye bank of a \\ universityhospital
}

\author{
Lauro Augusto de Oliveira ${ }^{1}$ \\ Beatriz Soares Corrêa ${ }^{2}$ \\ Gildásio Castello de Almeida $\mathbf{J r}^{3}$ \\ Marta Teixeira Ferrari ${ }^{4}$ \\ Luiz Kazuo Kashiwabuchi ${ }^{5}$
}

Estudo realizado no Setor de Córneas e Moléstias Externas da Disciplina de Oftalmologia do Departamento de Especialidades Cirúrgicas da Faculdade de Medicina de São José do Rio Preto (FAMERP-SP).

Residente do terceiro ano do Departamento de Oftalmologia da Faculdade de Medicina de São José do Rio Preto (FAMERP-SP).

Ex-Residente do Departamento de Oftalmologia da Faculdade de Medicina de São José do Rio Preto (FAMERP-SP). Estagiária do setor de Lente de Contato da Universidade Federal de São Paulo (UNIFESP). 3 Assistente do Setor de Córnea e Moléstias Externas do Departamento de Oftalmologia da Faculdade de Medicina de São José do Rio Preto, Doutor em Oftalmologia pela Faculdade de Medicina de Ribeirão Preto - USP.

${ }^{4}$ Chefe do Setor de Córnea e Moléstias Externas do Departamento de Oftalmologia da Faculdade de Medicina de São José do Rio Preto (FAMERP). Mestre em Medicina Interna pela (FAMERP - SP).

${ }_{5}^{5}$ Chefe do serviço de Oftalmologia da Faculdade de Medicina de São José do Rio Preto (FAMERP). Doutor em Oftalmologia pela Universidade Federal de Minas Gerais (UFMG).

Endereço para correspondência:Rua Abdo Muanis, n 1101 São José do Rio Preto (SP) CEP 15090-140 E-mail: laopadilha@hotmail.com.

Recebido para publicação em 18.07.2002 Aceito para publicação em 06.03.2003

Nota Editorial: Pela análise deste trabalho e por sua anuência na divulgação desta nota, agradecemos ao Dr. Paulo Ricardo de Oliveira.

\begin{tabular}{|l|}
\hline RESUMO \\
\hline Objetivo: Avaliar como a implantação do sistema de lista única para \\
transplantes de córnea influenciou um Banco de Olhos vinculado a um \\
hospital escola. Analisar sua interferência nas córneas (captação e desti- \\
no), no número de transplantes realizados e também na média de tempo de \\
espera pela cirurgia. Métodos: Foi realizado estudo retrospectivo, ava- \\
liando os prontuários dos pacientes submetidos a ceratoplastia penetran- \\
te e também os dados do Banco de Olhos da Faculdade de Medicina de São \\
José do Rio Preto - SP. O estudo comparou dados relativos ao fun- \\
cionamento do serviço por um ano antes e após a criação da lista única. \\
Resultados: O número de cirurgias aumentou de 60 para 92 cirurgias. A \\
média mensal de córneas retiradas aumentou de $13,83 \pm 6,57$ para $18,16 \pm$ \\
4,80 (p=0,07). O número de córneas enviadas por esta instituição foi maior \\
que o número de córneas recebidas de outros serviços (p=0,003). Não \\
houve diferença significativa entre o tempo de espera pela cirurgia antes \\
eapós a criação da filaúnica (desconsiderando o período de cadastramento). \\
Conclusões: Este Banco de Olhos funcionou como fornecedor de córneas \\
para outras instituições. Após seu primeiro ano de funcionamento, a \\
implantação da listaúnica não alterou o tempo de espera dos pacientes pela \\
cirurgia. Apesar disso, evidenciou-se uma tendência à homogeneização \\
do tempo de espera pela ceratoplastia penetrante entre os pacientes. \\
\hline
\end{tabular}

Descritores: Ceratoplastia penetrante; Banco de olhos; Tempos de espera; Listas de espera; Obtenção de órgãos; Doadores de tecidos; Transplante de córnea

\section{INTRODUÇ̃̃̃O}

Apesar da ceratoplastia penetrante ter se tornado o procedimento mais comum em relação aos transplantes ${ }^{(1-2)}$, ainda existem milhares de pessoas no Brasil à espera de uma córnea ${ }^{(3)}$. Este quadro impressiona, pois o tempo de espera para a cirurgia algumas vezes supera a sobrevida do paciente candidato ao transplante ${ }^{(3-4)}$.

Inúmeros são os obstáculos que atrapalham a progressão do número de transplantes realizados, sendo o sistema de doação - captação um dos mais relevantes $^{(3,5-7)}$. Diante dessa situação a Secretaria da Saúde do Estado de São Paulo vem, ao longo dos anos, se esforçando para proporcionar um aumento do número de doações e conseqüentemente do número de transplantes. Para tal já foram criados as Organizações de Procura de Órgãos (OPO) ${ }^{(8)}$ e o Sistema de Lista Única para Transplantes de Córnea - Organização e Procura de Córneas (OPC) ${ }^{(9)}$, pelo qual o Ministério da Saúde passou a normatizar os Bancos de Olhos (BO). O Estado de São Paulo foi dividido em dez regionais, cada uma tendo sua OPC e sua lista única para transplantes de córnea. 
O objetivo deste estudo foi verificar como a criação da OPC influenciou o BO do Hospital de Base (HB) da Faculdade de Medicina de São José do Rio Preto (FAMERP) em relação às córneas (doação, captação e destino), analisar sua interferência em relação ao número de transplantes realizados e também sobre a média de tempo de espera pela cirurgia antes e após a criação da OPC.

\section{MÉTODOS}

Foram avaliados, retrospectivamente, os prontuários dos pacientes submetidos ao transplante de córnea e também os dados da OPC - Hospital de Base - São José do Rio Preto. Esses dados continham informações do BO sobre captação e destino das córneas durante o período de 01/05/1999 até 01/05/2001. O Termo Aditivo 04/98 da Secretaria da Saúde do Estado de São Paulo referente à criação e a implantação das OPCs entrou em vigor em $01 / 05 / 2000^{(9)}$, portanto este trabalho comparou os dados relativos à nossa experiência por um ano antes e após a criação da lista única.

O BO - HB de São José do Rio Preto dispõe de um programa de captação, constituído por uma equipe composta por médicos, enfermeiros, psicólogos e assistentes sociais, que trabalham em regime de plantão integral. Essa equipe é responsável pela abordagem da família, seleção, captação e preservação das córneas. Os critérios de seleção, captação e preservação das mesmas obedecem às normas atuais da "Eye Bank Association of America" (EBAA) ${ }^{(10)}$. A enucleação é efetuada após o consentimento da família, sendo feita de maneira asséptica e no prazo máximo de seis horas após o óbito. O sangue do doador é colhido e encaminhado ao laboratório para testes sorológicos de HIV e hepatites B e C. A preparação das córneas é feita imediatamente após a enucleação do bulbo ocular e, uma vez confirmada a integridade do tecido corneano biomicroscopicamente, o mesmo é preservado em OPTSOL ${ }^{\mathrm{TM}}$-GS - "Corneal Storage Media" (Bausch \& Lomb, Irvine, EUA), com o objetivo de ser usado no prazo médio de 72 horas. Esta sistematização do BO foi a mesma durante todo o tempo do estudo.

Foram estudados: o número de transplantes realizados, a média de tempo de espera entre a inscrição do paciente e a realização da cirurgia, o número de córneas captadas e disponíveis para este serviço, bem como o de córneas recebidas e enviadas pelo $\mathrm{BO}$ - HB.

Excluíram-se os casos dos pacientes que foram inscritos como prioridade (13 pacientes), pois não obedeciam a ordem cronológica de convocação para a cirurgia. Também se excluíram os casos em que os dados estavam incompletos em relação aos quesitos do protocolo (3 pacientes).

Com a criação das OPCs houve um período de cadastramento dos pacientes para que eles fossem inscritos na lista única de cada uma das regionais, mantendo como parâmetro a data de inscrição no BO de origem. Este fato aumentou o tempo de espera pela cirurgia dos primeiros pacientes da lista do $\mathrm{BO}$ - HB, uma vez que pacientes de outros $\mathrm{BO}$ e outros municípios passaram a integrar a lista da OPC Rio Preto. Os cálculos do tempo de espera foram feitos dividindo os pacientes em 2 grupos: antes da criação da OPC e após a criação da OPC. Para diminuir a interferência do cadastramento inicial, calculou-se também o tempo de espera pela cirurgia desconsiderando os primeiros 22 pacientes inscritos na lista do BO HB. Este novo cálculo baseou-se no fato de que estes pacientes esperaram pelo transplante por um tempo consideravelmente maior do que os demais, devido à entrada simultânea de vários pacientes com datas de cadastramento distintas.

Para a análise estatística foi utilizado o teste t comparando o tempo de espera do transplante, antes e após a implantação da OPC, bem como o número de córneas retiradas e disponíveis para o serviço. $O$ teste $t$ pareado também foi utilizado para a avaliação do intercâmbio de córneas. Para a avaliação dos resultados obtidos sobre o tempo de espera pelo transplante foi realizada análise de regressão linear. Para todos os testes adotou-se um nível de significância $\alpha=0,05$.

\section{RESULTADOS}

Durante o período do estudo foram realizadas 152 ceratoplastias penetrantes. Houve um aumento de 60 para 92 cirurgias no primeiro ano de funcionamento da lista única para transplantes de córneas. Com a implantação da OPC notou-se um aumento da média mensal de córneas retiradas de 13,83 \pm 6,57 para $18,16 \pm 4,80(\mathrm{p}=0,07)$. Houve também um aumento da média mensal de córneas disponíveis de $6,58 \pm 3,98$ para $8,91 \pm$ $4,71(\mathrm{p}=0,20)$ (Tabela 1$)$.

Com o intercâmbio de córneas iniciado após a criação das OPCs, percebemos que o $\mathrm{BO}$ - $\mathrm{HB}$ funcionou como fornecedor de córneas para outros BOs. Enviou 72 córneas enquanto recebeu apenas 10 (Gráfico 1 ).

A média de tempo de espera pela cirurgia aumentou de $110,74 \pm 44,00$ para $134,83 \pm 59,39$ dias após a criação da OPC. Desconsiderando o período de cadastramento dos pacientes

\begin{tabular}{|c|c|c|c|}
\hline Banco de Olhos & $\begin{array}{l}\text { Número de ceratoplastias } \\
\text { penetrantes realizadas }\end{array}$ & $\begin{array}{l}\text { Média mensal de } \\
\text { córneas retiradas }\end{array}$ & $\begin{array}{l}\text { Média mensal de } \\
\text { córneas disponíveis }\end{array}$ \\
\hline Antes da criação da OPC & 60 & $13,83 \pm 6,57$ & $6,58 \pm 3,98$ \\
\hline Após a criação da OPC & 92 & $18,16 \pm 4,80$ & $8,91 \pm 4,71$ \\
\hline Teste t não pareado & & $p=0,07$ & $p=0,20$ \\
\hline
\end{tabular}




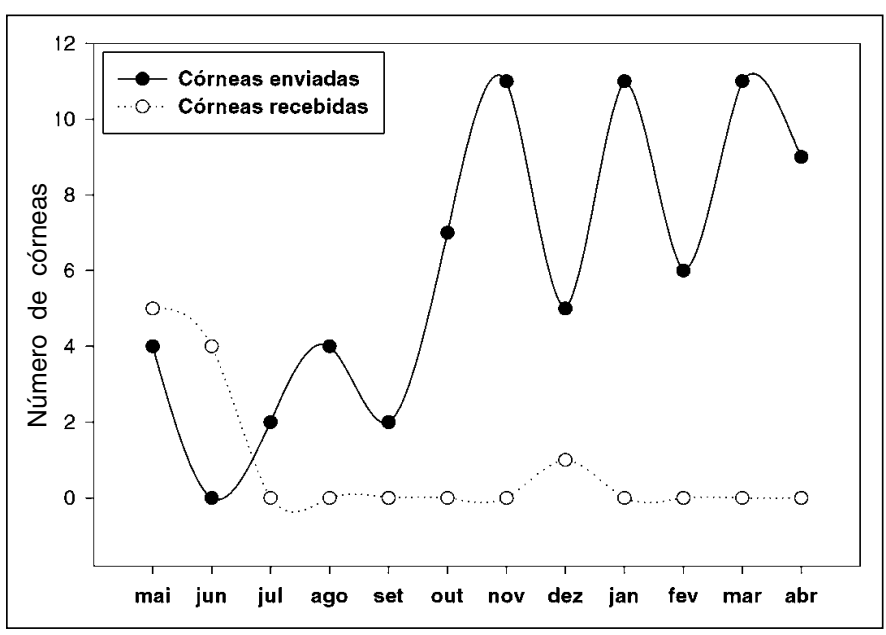

Gráfico 1 - Número de córnas enviadas e recebidas pelo Banco do Olhos do Hospital de Base após a criação da OCP no periodo de maio de 2000 a abril de 2001

na lista única percebemos que não houve diferença significativa entre os tempos de espera pelo transplante antes e após a criação da OPC (Gráfico 2).

$\mathrm{O}$ estudo de regressão linear realizado com os pacientes divididos em três grupos: antes da criação da OPC; depois da criação da OPC e após a criação da OPC desconsiderando o período de cadastramento evidenciou uma tendência à homogeneização do tempo de espera dos pacientes pelo transplante após a criação da OPC (Gráficos 3,4 e 5).

\section{DISCUSSÃO}

O número de transplantes realizados aumentou de 60 para 92 após a criação da OPC (Tabela 1). Estes dados estão relaciona-

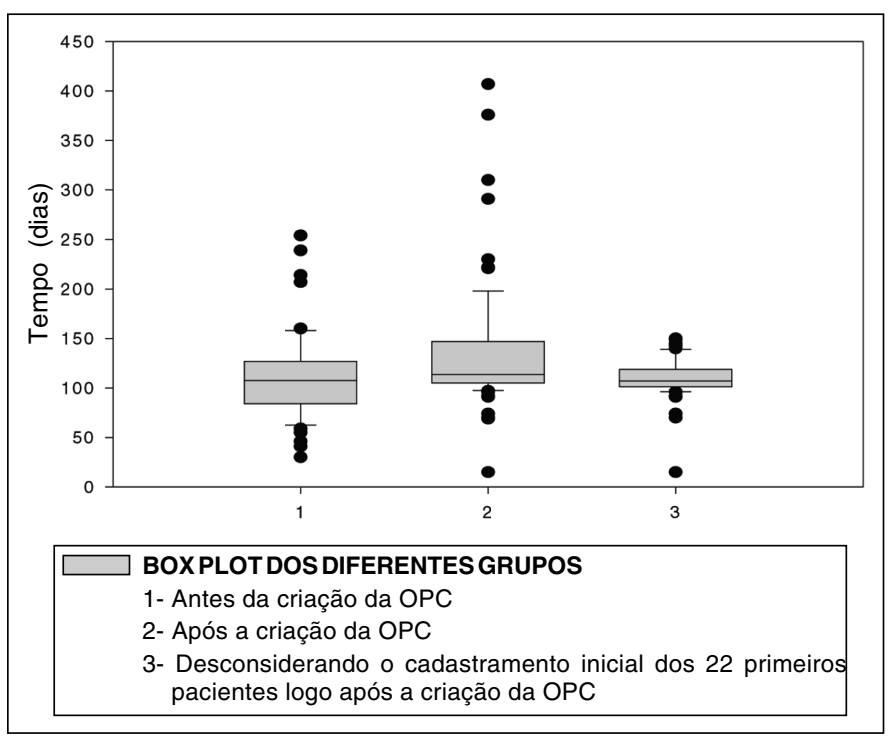

Gráfico 2 - "Box Plot" do tempo de espera pela cirurgia antes e após a criação da OPC

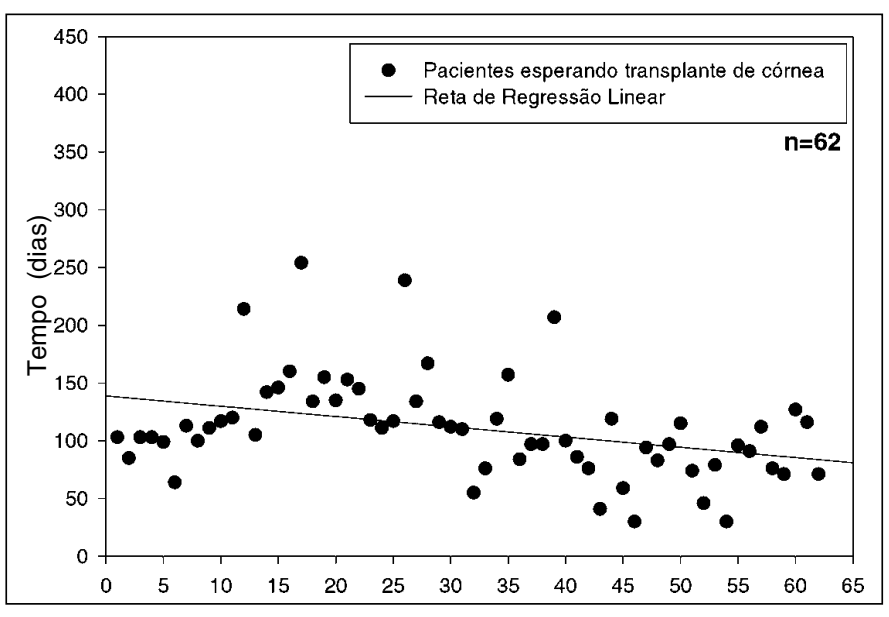

Gráfico 3 - Tempo de espera em dias pela cirurgia antes da criação da OPC

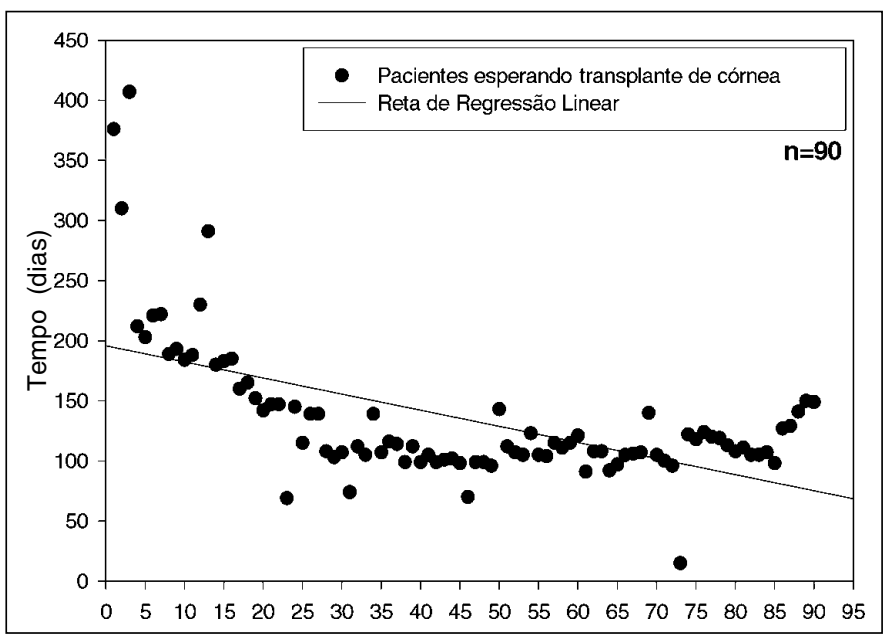

Gráfico 4 - Tempo de espera em dias pela cirurgia após a criação da OPC

dos com o número de córneas captadas pelo serviço e também com o número de córneas disponíveis. Como o BO - HB funcionou em tempo integral e a sistematização da captação de córneas foi a mesma durante o estudo, não encontramos um fator evidente que justificasse o aumento da média mensal de córneas captadas após a criação da OPC. Aumentou de 13,87 \pm 6,57 para $18,16 \pm 4,80$ após a criação da $\mathrm{OPC}(\mathrm{p}=0,07)$. O número de córneas disponíveis reflete diretamente o número de transplantes realizados. Apesar de não significativo $(\mathrm{p}=0,20)$, houve um aumento da média mensal de córneas disponíveis para o $\mathrm{BO}$ - HB após a criação da OPC (Tabela 1). Isto colaborou para o aumento do número de transplantes realizados.

$\mathrm{O}$ BO - HB funcionou como fornecedor de córneas para outros serviços no primeiro ano de implantação do sistema de lista única (Gráfico 1). Antes da criação da OPC as córneas eram captadas e preservadas para utilização única e exclusiva dos pacientes da própria instituição. Após a viabilização da OPC iniciou-se o intercâmbio de córneas entre os Bancos de Olhos. As córneas dos vários Bancos de Olhos passaram a ser 


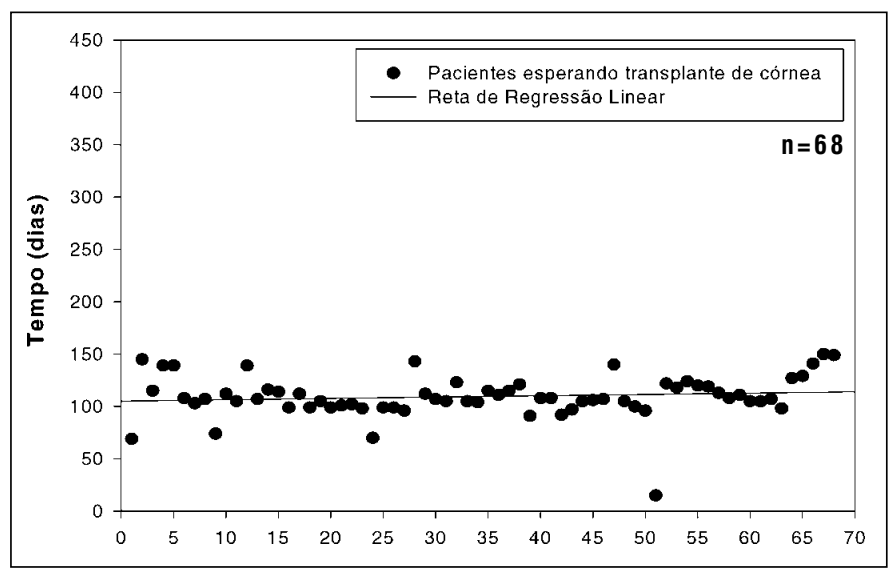

Gráfico 5 - Tempo de espera em dias pela cirurgia após a criação da OPC, desconsiderando período de cadastramento inicial

enviadas para a instituição que continha o primeiro paciente da lista. Caso este paciente fosse inscrito pela própria instituição que realizou a captação, a córnea permaneceria no mesmo BO. Assim, o BO - HB, no seu primeiro ano de funcionamento, enviou mais córneas do que recebeu $(\mathrm{p}=0,003)$.

Os resultados que comparam a média de tempo de espera pela cirurgia antes e após a criação da OPC são contraditórios. Apesar do aumento do número de transplantes realizados e também do número de córneas captadas e disponíveis para o $\mathrm{BO}$ - HB, houve um aumento no tempo de espera pela cirurgia após a criação da OPC. A média de tempo de espera pela cirurgia aumentou de $110,74 \pm 44,00$ dias para 134,83 $\pm 59,39$ dias após a criação da OPC $(\mathrm{p}=0,007)$.

Alguns comentários podem justificar tal contradição. Notificamos que o $\mathrm{BO}$ - HB funcionava de forma independente e autônoma antes da criação da OPC. Assim, havia liberdade para manipular os pacientes inscritos nesta instituição. Isto não ocorria beneficiando ou prejudicando qualquer paciente em relação a sua posição na lista, mas sim, viabilizando uma córnea disponível de maneira mais rápida e menos burocrática. Por exemplo, caso o primeiro paciente da fila estivesse inapto para a cirurgia, havia liberdade para convocar o próximo, de acordo com a conveniência do serviço. Agindo desta maneira havia interferência no tempo de espera pela cirurgia destes pacientes. Proporcionava-se uma heterogeneidade notável entre o tempo de espera de um e outro paciente. Com a implantação da OPC esta liberdade deixou de existir. As córneas passaram a ser destinadas para os pacientes por uma central de transplantes. Portanto, ao contrário do que ocorria, iniciouse uma tendência à homogeneização do tempo de espera pelo transplante entre os pacientes. Analisando o Gráfico 2 percebemos claramente como há uma maior condensação dos pacientes do grupo 3 (pacientes após a criação da OPC, desconsiderando o cadastramento dos 22 primeiros) em relação à média, quando comparados com os pacientes do grupo 1(pacientes antes da criação da OPC). Da mesma forma observamos a diferença entre os pontos anômalos que representam os pacientes dos dois grupos.
Outro fato relevante que interferiu no tempo de espera pela cirurgia foi o processo de cadastramento dos pacientes quando do início da lista única. Nessa época, vários pacientes que se encontravam em posições privilegiadas na lista do $\mathrm{BO}$ - HB perderam lugares quando entraram na lista única e ganharam um tempo adicional de espera pela cirurgia. Fato percebido observando os pontos anômalos que representam os pacientes do grupo 2 (pacientes após a criação da OPC) (Gráfico 2).

Portanto, uma vez desconsiderada a fase de cadastramento (22 primeiros pacientes), não há diferença significativa entre o tempo de espera pelo transplante antes (110,74 dias) e após (109,52 dias) a criação da OPC $(p=0,91)$.

Felizmente, desconsiderando ou não o período de cadastramento, a média de tempo de espera neste serviço se assemelha a dos centros europeus, onde o tempo médio de espera foi de 5,5 meses (1-16 meses $)^{(11)}$.

A análise de regressão linear feita com os pacientes antes da criação da OPC (Gráfico 3) mostra dados interessantes. É uma reta decrescente e mostra a heterogeneidade do tempo de espera entre os pacientes. Esta reta definida pela função $f(x)=$ $138,76-0,88 x$, deixa claro que havia uma tendência a zerar a lista de transplantes de córnea do BO - HB caso não fosse implantado o sistema de lista única. Permite ainda estimar que isto ocorreria por volta do transplante de número 158 a partir do início do estudo.

A regressão linear feita com os pacientes após a criação da OPC também mostra uma reta decrescente (Gráfico 4). Observamos uma heterogeneidade inicial com valores elevados do tempo de espera pela cirurgia. Estes valores discrepantes coincidem com o período de cadastramento. Atribuímos a este período a responsabilidade por delinear uma reta $(\mathrm{f}(\mathrm{x})=195,79$ - 1,33x) com um coeficiente decrescente maior que o da reta do Gráfico 3. Daí surge a necessidade de analisarmos o comportamento desta reta desconsiderando o cadastramento dos primeiros 22 pacientes. Assim, tivemos uma reta definida por $f(x)=105,14+0,12 x$ que caracteriza uma ligeira tendência crescente ou à homogeneização do tempo de espera pela cirurgia (Gráfico 5). Observamos a homogeneidade dos tempos de espera pela cirurgia dos vários pacientes de acordo com a sua proximidade com a reta.

Como citado anteriormente, pouco pode se dizer em relação ao aumento do número de captações após a criação da OPC. Acreditamos que este dado possa ser justificado pela melhor conscientização dos médicos, em especial dos intensivistas, no sentido de informar o serviço de captação sobre potenciais doadores. Também é provável que haja um nível de credibilidade e confiança maior, por parte da população, sobre o funcionamento e responsabilidade dos Bancos de Olhos em relação às córneas, após a criação das OPCs.

\section{CONCLUSÃO}

Baseado nos dados encontrados conclui-se que a criação da OPC, neste primeiro ano de funcionamento, demonstrou 
uma forte tendência à homogeneização do tempo de espera pelo transplante. Uma vez mantida essa tendência poderá se estimar com boa margem de segurança o tempo provável de espera de cada paciente na lista.

Observou-se que houve uma evolução do sistema de captação-doação deste Banco de Olhos, devido ao aumento do número de córneas captadas e cirurgias realizadas após a criação das OPCs.

Ressalta-se também o papel relevante do BO - HB como fornecedor de córneas para outros Bancos de Olhos durante seu primeiro ano de funcionamento.

\section{ABSTRACT}

Purpose: To evaluate how the implantation of a single regional list for corneal transplants influenced an eye bank of a university hospital. To analyze the influence of the regional list on the corneas (procurement and destiny), the number of transplantations made and the average waiting time for surgery. Methods: A retrospective study was made to compare the records of patients who underwent penetrating keratoplasty and also the data of the eye bank of the Medical School of São José do Rio Preto, São Paulo, Brazil. The study compared the data of the service one year before and one year after the creation of the single regional list. Results: The number of transplantations increased from 60 to 92 procedures. The monthly average of directly acquired corneas increased from $13.83+6.57$ to $18.16+4.8(\mathrm{p}$-value $=0.07)$. The number of corneas sent to other institutions was greater than the number of corneas received from other eye banks ( $p$-value $=0.003$ ). There was no significant difference between the waiting time for surgery before and after the creation of the list except for the period of adaptation. Conclusions: This eye bank served as a supplier of corneas for other institutions. By the end of the first year of operation of the single regional list, there was no alteration in the waiting time as compared to that before its creation. Nonetheless, a tendency of homogenization in the waiting time for penetrating keratoplasty among the patients was observed.

Keywords: Keratoplasty penetrating; Eye Banks; Waiting periods; Waiting lists; Organ procurement; Tissue donors; Corneal transplantation

\section{REFERÊNCIAS}

1. Freitas JAH, Soranz JE, Rivogatti Junior V, Barbosa ML, Hirashima DE, Felberg S. Ceratoplastia penetrante. Experiência de um ano de um Banco de Olhos vinculado a um Hospital Escola. Rev Bras Oftalmol 1997;56:749-54.

2. Report of the organ transplantation panel. Corneal transplantation. JAMA 1988;259:719-22.

3. Alves MR, Crestana FP, Kanatani R, Cresta FB, José NK. Doação de córnea: opinião e conhecimento de médicos intensivistas do complexo Hospital das Clínicas da Faculdade de Medicina da Universidade de São Paulo. Rev Méd (São Paulo) 1997;76:315-9.

4. Nenno C, Abel RJ. Cornea transplantation statistics in the United States. Refract Cornea Surg 1991;7:467-8.

5. Pestana JO, Vaz ML, Delmonte CA, Cavecchia SR, Pacheco M, Piveta VM, et al. Estimativa do número de potenciais doadores de órgãos na cidade de São Paulo. Rev Assoc Med Brasil 1992;38:97-100.

6. Siminoff LA, Arnold RM, Caplan AL. Health care professional attitudes toward donation: effect on practice and procurement. J Trauma 1995;39:553-9.

7. First MR. Transplantation in the nineties. Transplantation 1992;53:1-11.

8. São Paulo. Secretaria de Estado da Saúde. Resolução SS - 103 de 01/08/97. Dispõe sobre a estrutura organizacional e operacional do Sistema Estadual de Transplantes de São Paulo. Diário Oficial do Estado, São Paulo, 05 fev 1997.

9. São Paulo. Secretaria de Estado da Saúde. Termo Aditivo 04/98; Processo $n^{\circ}$ 001/0001/001.511/98; Secretaria de Estado da Saúde - Fundação Faculdade de Medicina de São José do Rio Preto; "Relator; José da Silva Guedes; 2 de julho de 1998. Diário Oficial do Estado, São Paulo, 4 jun 1998. n¹26, p.51”.

10. Eye Bank Association of America. Administrative manual. Medical standards. Washington DC: EBAA; 1992.

11. Beekhuis WH. Current clinician's opinios on risk factors in corneal grafting: results of a surgery among surgeons in the eurotransplant area. Cornea $1995 ; 14: 39-42$.

\title{
ABO ELETRÔNICO
}

\author{
Novo site
}

\section{Acesso: http://www.abonet.com.br}

